\title{
Comércio Eletrônico: Fazendo Negócios por meio da Internet
}

\author{
Eduardo Henrique Diniz
}

\section{RESUMO}

O comércio eletrônico favorece novas formas de obtenção de ganhos de competitividade nos negócios, interferindo na cadeia de valor adicionado a produtos e serviços dirigidos ao consumidor ou em transações entre empresas. Sendo global em sua essência, o comércio eletrônico está expandindo-se rapidamente em todo o mundo; no entanto essa velocidade e o alcance do comércio eletrônico enfrentam obstáculos de vários tipos. Existem obstáculos tecnológicos, culturais, organizacionais e estruturais para o pleno desenvolvimento do comércio eletrônico. Estas barreiras para o pleno desenvolvimento do comércio on-line devem ser também analisadas, para se entenderem mais claramente os possíveis efeitos no ambiente de negócios.

Palavras-chaves: comércio eletrônico; internet; EDI; limitações da TI.

\begin{abstract}
Electronic commerce has potential for levering business activities to add value to products and services, either in business-to-business or in business-to-customer transactions. Based on the Internet, which supports the global on-line business infrastructure, electronic commerce is growing fast all over the world. However, this speed and reach confront many obstacles. There are technological, cultural, organizational and structural barriers braking electronic commerce development. These barriers to develop the on-line commerce must be considered for a better understanding of the potential effects on business environment.
\end{abstract}

Key words: electronic commerce; internet; EDI; IT limitations. 


\section{Comércio Eletrónico}

O comércio eletrônico identifica o uso intensivo de Tecnologia da Informação (Beam e Segev, 1996) na mediação das relações entre consumidores e fornecedores. Para a melhor compreensão das vantagens e riscos do comércio eletrônico, no entanto, é útil a sua divisão em dois blocos principais.

O primeiro bloco indica as atividades relativas a transações entre empresas que compram e vendem produtos entre si. Este grupo se caracteriza por um número relativamente baixo de transações de alto valor financeiro. O segundo bloco, das transações entre empresas e consumidores finais, se caracteriza por alto volume relativo de transações com baixo valor financeiro envolvido em cada uma delas.

Nas transações empresa-empresa, o Electronic Data Interchange (EDI) é o exemplo mais conhecido (Sokol, 1989). Apesar de já existir há algumas décadas, o EDI também está sendo afetado pela expansão da Internet.

Algumas experiências de utilização de EDI baseado na Internet têm sido estudadas com o objetivo de expandir as possibilidades desse tipo de tecnologia (Segev et al., 1995).

Na comparação da Internet com as outras alternativas de EDI, as questões custo e segurança são as mais ponderadas. O custo de implantação de EDI pela Internet é baixo, enquanto o das redes privadas é alto. Para se ter uma comparação, num estudo feito no Bank of America pôde ser observado que uma transação feita pela Internet chega a ser entre duas e três ordens de grandeza menor do que a mesma transação feita por uma VAN.

Em compensação, em termos de segurança, o uso de EDI pela Internet não tem ainda os mesmos níveis de confiança dos usuários que têm as redes privadas. Se numa rede privada existe alto grau de controle das informações que nela trafegam, na Internet a situação é um tanto diferente. Apesar disso, o crescente interesse comercial na Internet começa a influenciar o desenvolvimento de recursos para aumentar a confiabilidade e o grau de controle sobre as informações em trânsito, tais como tempo para entrega e priorização de mensagens.

Além de encriptação, outros dispositivos de segurança, como firewalls para controlar o acesso físico à rede, podem ser acrescentados ao sistema de EDI pela Internet. Para garantir todo o processo quanto a falhas humanas e naturais, os pontos de maior possibilidade de falhas podem adotar sistemas de redundância. 
Por tratar de área na qual as transações são mais estruturadas, o potencial da Internet em aplicações que envolvem transações entre empresas é muito grande; mas o lado do comércio eletrônico que mais têm atraído a atenção são as suas possibilidades de colocar empresas em contato com consumidores finais de qualquer lugar, a qualquer hora.

\section{O Valor do Comércio Eletrónico}

Para compreender o valor do comércio eletrônico, é preciso compará-lo com as formas de transações comerciais tradicionais e verificar como ele pode transformálas. A compreensão das possibilidades e limitações do comércio eletrônico ajuda a encontrar meios de melhorar a qualidade de um serviço ou de se desenvolverem mercados de outra forma inacessíveis. O comércio eletrônico também abre novas possibilidades de negócios que seriam impensáveis anteriormente.

Imaginar que o comércio eletrônico se restringe unicamente à venda direta de informações, serviços e produtos estreita a visão do impacto potencial sobre os negócios que a utilização comercial da Web pode oferecer a uma organização. Apesar da venda direta ser certamente a primeira forma de se pensar a obtenção de lucros numa relação entre consumidor-vendedor, a utilização da Web como veículo para o comércio eletrônico permite visualizar uma série de outras formas de adicionar valor a um negócio.

A visão do comércio eletrônico, como qualquer tipo de apoio de transações comerciais por meio do uso de infra-estrutura digital, tem a vantagem de englobar uma gama variada de utilizações da Web para favorecer ou incentivar transações comerciais.

Assim, o comércio eletrônico pode funcionar como instrumento de promoção (pré-venda), como novo canal de vendas de fato ou de atendimento ao cliente (pós-venda). Pode gerar economia na realização de transações e redução do ciclo de desenvolvimento dos produtos; a sua implementação deve promover um aprendizado organizacional e tecnológico indispensável para a sua aplicação efetiva. Além disso, se acredita (Bloch, Pigneur e Segev, 1996) que o comércio eletrônico ainda propicia o desenvolvimento de novos produtos e mesmo de novos modelos de negócio. 


\section{Barreiras e Desafios na Implantação do Comércio Eletrónico}

Um espantoso crescimento do comércio eletrônico nos próximos anos está sendo esperado por acadêmicos, especialistas e executivos (Hoffman, Novak e Chatterjee, 1995). Alguns apostam que os valores transacionados anualmente online atingirão dezenas de bilhões de dólares antes do fim da década. Mas para a plena efetivação do processo de compras on-line, barreiras tecnológicas, culturais e organizacionais devem ser transpostas, juntamente com o desenvolvimento de soluções que ajudem a superar algumas dificuldades estruturais do comércio eletrônico.

\section{Barreiras Tecnológicas}

Considerando o relacionamento empresa-consumidor, dois pontos são fundamentais para a evolução da tecnologia utilizada no comércio eletrônico: a disponibilidade da tecnologia e a sua facilidade de uso. Enquanto a disponibilidade está relacionada com acesso e custo da tecnologia, a sua facilidade de uso está relacionada com a evolução das interfaces de comunicação com os usuários.

Atualmente, para se ter acesso à Internet é necessário que se tenha à disposição computadores equipados com hardware específico para a interconexão, softwares apropriados para o gerenciamento e monitoração da comunicação e, no mínimo, uma linha telefônica comum que permita a interligação via computador com um provedor de acesso que viabiliza a conexão.

Se a utilização de computadores no ambiente doméstico continua crescendo, o que é fator positivo para o comércio eletrônico, é difícil dizer se apenas o ritmo desse crescimento será suficiente para consolidá-lo. De qualquer forma, a efetivação do comércio eletrônico vai demandar muito esforço tecnológico para colocar os consumidores em contato com a tecnologia necessária para a sua utilização.

Não faltam empresas dispostas a investir nesse esforço. O acesso à Web via TV está diretamente relacionado com o esforço de tornar o comércio eletrônico mais acessível. A WebTV é projeto no qual estão empenhados tradicionais fabricantes de aparelhos de TV, empresas da indústria da computação e grandes corporações de comunicação.

Por causa da Internet, o próprio conceito de PC está sendo discutido. Se antes se julgava que os computadores tinham de ser cada vez mais potentes, dando maior poder de processamento ao usuário, agora se discute um novo tipo de computador, que deve primar pela simplicidade. Esse novo tipo de computador, o Network Computer, ou NC, está sendo considerado seriamente por todas as grandes empresas de informática. 
Pelo lado das telecomunicações, a Internet também está causando forte impacto. Por causa do longo tempo médio que um usuário da Internet permanece conectado, companhias telefônicas por todo o mundo estão alegando elevado crescimento da demanda por serviços de telefonia. Se o aumento de demanda pode trazer aumento de receita, a velocidade de crescimento dessa demanda tem dificultado a operação das companhias telefônicas, que não conseguem responder com investimentos na mesma proporção.

Além disso, o aumento de demanda está gerando discussões sobre as formas de cobrança de tarifas telefônicas. Companhias telefônicas (Telecom, 1996) já anunciam que vão operar via Web, utilizando a tecnologia de conexão que mantém as características das ligações via Internet. Isso pode significar o fim da cobrança das ligações interurbanas, uma vez que as conexões da Internet são geralmente feitas via ligações locais.

Caso esse ritmo de demanda prevaleça, em pouco tempo os serviços telefônicos em muitos países estarão muito próximos da saturação. Existirão linhas telefônicas disponíveis para atender algumas das previsões mais otimistas (Negroponte, 1995)? Para tentar atender à demanda por mais e melhores conexões à Internet, as empresas que operam sistemas de TV a cabo estão entrando na disputa com as companhias telefônicas.

É de se esperar que o aumento da concorrência na área das telecomunicações contribua para facilitar o acesso ao comércio eletrônico; mas a concorrência, nesse caso, esbarra em alguns problemas legais de regulamentação. Há países nos quais operadoras de TV a cabo sofrem restrições para atuar como provedoras desse tipo de serviço e as telecomunicações estão sujeitas a um controle, quando não a uma administração direta muito forte por parte dos Governos. De qualquer forma, o mercado de provedores de acesso à Internet ainda está em estágio de consolidação (Gupta, Stahl e Whinston, 1996).

As alternativas para aumentar a possibilidade de acesso à Internet continuam a aparecer. Nos EUA, uma recente regulamentação da Federal Communications Commission (FCC), órgão que regula as comunicações, está facilitando a criação de redes sem fio de alcance limitado, independentes dos sistemas de telefonia, para facilitar o estabelecimento de conexões mais baratas à Internet (The New York Times, 1997).

A disponibilidade de recursos tecnológicos não pode prescindir da evolução da facilidade de uso desses mesmos recursos. Apesar de a Web ter simplificado imensamente a utilização da Internet, ela ainda é essencialmente uma rede de computadores. Os computadores ainda não são tão simples de se utilizar quanto uma TV ou um telefone, por exemplo. Esse aspecto é especialmente crítico, se for conside- 
rada a multiplicidade de serviços que podem ser implementados para o atendimento on-line de consumidores e que devem ser programados pelos próprios usuários. Deve-se esperar que a velocidade de adesão aos sistemas de comércio eletrônico esteja também atrelada ao nível de complexidade dos mesmos.

O desenvolvimento de agentes virtuais inteligentes e outras tecnologias interativas contribuirá para a ampliação do uso do comércio eletrônico, facilitando a interação dinâmica entre computadores. Pequenas aplicações, ou applets, serão enviadas dos computadores-clientes para a rede, possibilitando grande aumento de interatividade na utilização da Web. Esses applets, instruídos para auxiliar o usuário na busca de informações de maneira rica e precisa, farão o papel de agentes inteligentes; mas tais recursos estão ainda em estágio muito embrionário.

No nível das interações empresa-empresa, o comércio eletrônico também precisa superar dificuldades, para que seja amplamente adotado. A principal dificuldade para o aumento das transações entre empresas ainda está na confiabilidade dos sistemas de segurança adotados. Apesar de ser possível adotar na Internet sistemas que teoricamente tenham nível de segurança equivalente aos sistemas adotados em outros veículos disponíveis para as empresas trocarem informações, as constantes notícias de invasão de sistemas de grandes empresas, e até de departamentos de segurança de Governos, ajudam a reforçar, na opinião pública em geral, a idéia de que a Internet não é segura.

Outro ponto que dificulta a implantação de um nível de segurança mais elevado nas transações via Internet está em exigências do próprio Governo dos EUA. Preocupado em limitar a inviolabilidade da troca de informações entre terroristas e outros grupos que possam, de alguma forma, se utilizar da Internet para atividades ilegais, o Governo norte-americano está exigindo uma limitação no número de bits de encriptação das mensagens. Como a limitação da encriptação está diretamente relacionada ao aumento da segurança na Internet (Levy, 1996), diversas empresas estão tentando persuadir o Governo norte-americano da necessidade de ampliar a encriptação e da inocuidade das restrições que estão sendo impostas, uma vez que em outros países não existe tal legislação restritiva.

\section{Barreiras Culturais}

É fato que o uso da Internet está fortemente concentrado nos EUA, embora evoluindo rapidamente na Europa e em outros continentes. De qualquer forma, um dos componentes do sucesso do comércio eletrônico está relacionado com hábitos de consumo comuns entre os norte-americanos, como as compras por meio de catálogos e via TV. É de se esperar que em países com diferentes hábitos de consumo, a implantação de sistemas de comércio eletrônico vá ocorrer em ritmo diferente. 
Outras barreiras, como a língua e peculiaridades culturais, são mais difíceis de serem avaliadas, mas também podem constituir obstáculos para a ampla disseminação de transações comerciais on-line em nível global, embora não invalidem a utilização do comércio eletrônico em regiões geograficamente delimitadas. Isto significa que não basta alguém ter acesso aos recursos da Internet para poder fazer aquisições em qualquer lugar do planeta, embora isso seja virtualmente possível. Assim, a acomodação de diferenças culturais, fenômeno intrínseco às atividades de comercialização global on-line, deve ser levada em conta no desenvolvimento dos sistemas de comércio eletrônico.

Há que se considerar que a adoção dos sistemas de comércio eletrônico estará associada ao tipo de produto fornecido (Steinfield, Kraut e Plummer, 1995). Os processos de compra diferem não apenas segundo o perfil do consumidor, mas também para cada tipo específico de produto.

Mesmo considerando produtos, cujo conteúdo está vinculado à informação neles contida, como no caso de livros e discos, o hábito de compra está profundamente associado à apreciação física do produto que se quer adquirir. A transmissão digital on-line das informações contidas nesse tipo de produto, apesar de ser viável tecnicamente e de estar sendo acreditada por muitos como uma das possibilidades mais interessantes do comércio eletrônico, depende de uma aceitação ampla da massa de consumidores que, mesmo tendo a possibilidade de selecionar os produtos eletronicamente, tem o desejo de possuir fisicamente o objeto de sua compra.

A possibilidade de aceitação do comércio eletrônico tem implicações diferentes, quando se trata de produtos como software, por exemplo. A necessidade de colocar em funcionamento toda a estrutura do comércio eletrônico tem demandado a multiplicação de programas e de empresas, que apresentam inúmeras novas alternativas para facilitar a utilização da Internet. Como a maioria desses softwares está sendo distribuída pela própria Internet, novos padrões de comercialização de programas de computador se estão consolidando.

Ao se transformar no veículo preferencial para a comercialização de softwares, a Internet pode transformar também a idéia por trás do fornecimento desse tipo de produto: a venda se poderia transformar em locação. De fato, por meio do comércio eletrônico a mercadoria software pode estar sujeita a este tipo de distribuição caracterizada como venda por assinatura e com tempo de utilização limitado.

Uma barreira que impede a adoção ampla desse tipo de estratégia atualmente é o tempo gasto para download de softwares via Internet; mas mesmo que a tecnologia evolua rapidamente e possa tornar-se mais flexível, a idéia de locação de software ainda vai encontrar obstáculos. Apesar de que as empresas de software insistem 
que, quando se compra um pacote com um programa, se está adquirindo não um programa de fato, mas uma licença para a sua utilização, essa mensagem não é claramente assimilada. Esse problema está relacionado com a satisfação do desejo de posse sobre o produto.

Além dos processos de compra, a garantia de privacidade é também fator crítico para ajudar a desenvolver no consumidor certo nível de confiança nos sistemas de comércio eletrônico. Se por um lado o sistema pode melhor servir ao cliente quanto mais informações sobre ele lhe forem disponibilizadas, é patente que há resistências para essa coleta de informações por parte dos consumidores. Principalmente quando se considera que o público em geral ainda acha que computadores podem servir para exercer maior controle sobre as atividades dos indivíduos.

Se os consumidores não têm confiança suficiente no sistema para fornecer seus dados, não é possível levantar informações demográficas, nem os padrões de compra, nem as necessidades específicas do mercado, que foram apontados anteriormente como importantes para as estratégias do comércio eletrônico. Para superar a falta de confiança, há apenas a percepção da melhoria significativa no processo de compra, proporcionado pelo comércio eletrônico (por exemplo, produtos ajustados a necessidades individuais), ou a adoção de estratégias que ofereçam incentivos (descontos ou brindes) aos consumidores em troca de informações.

A falta de confiança dos consumidores também se manifesta em questões relativas à segurança dos sistemas de comércio eletrônico. Existe a percepção entre os consumidores de que as redes de computadores estão sujeitas a ataques constantes de pessoas ou grupos interessados em roubar ou adulterar informações. Apesar de essa percepção poder ser considerada exagerada (Kline, 1995), ela existe e deve ser levada em conta, para que o uso dos sistemas de comércio eletrônico possa ser expandido. Além disso, a facilidade de se criarem empresas em qualquer lugar e vender produtos para o mundo todo implica o risco de um consumidor transacionar com uma empresa virtual, que desaparece assim que recolhe o seu dinheiro.

Para assegurar a defesa da privacidade e segurança dos consumidores on-line e garantir o desenvolvimento do comércio eletrônico num ambiente de confiança, empresas nos EUA estão patrocinando organizações (Eletronic Frontier Foundation e eTrust) com a missão de estabelecer padrões de segurança e autenticação. A atuação dessas organizações se baseia no fato de que os consumidores têm o direito de serem informados sobre as consequiências que uma transação online pode trazer à sua privacidade e segurança, antes de realizá-la. 


\section{Barreiras Organizacionais}

Se pelo lado dos consumidores existem barreiras culturais, pelo lado das empresas devem ser consideradas as barreiras organizacionais, que podem representar obstáculos para o crescimento do comércio eletrônico (Ware et al., 1998). Em primeiro lugar, as empresas devem estar cientes das implicações administrativas, para que criem, giram e obtenham os benefícios da adoção de sistemas de comércio eletrônico. A definição clara de uma estratégia de negócios deve existir, para integrar a nova tecnologia à organização.

A introdução de sistemas de comércio eletrônico pode implicar a necessidade de se redefinir alguns processos organizacionais para torná-los perfeitamente integrados com os sistemas de solicitação de pedidos e serviços feitos pelos consumidores. Essa integração vai exigir esforços de integração de sistemas e plataformas, especialmente nos casos em que os sistemas de controle dos processos internos pré-existentes foram concebidos sobre tecnologias e filosofias de desenvolvimento diferentes daquelas que predominam no ambiente da Internet e da Web.

A tecnologia por si só não é suficiente para alavancar um negócio na direção de garantir retorno na adoção de sistemas de comércio eletrônico. É preciso que seja criada uma vantagem competitiva sustentável, que garanta certa fidelidade do cliente para com a empresa. A qualidade do serviço oferecido e o nível de relacionamento entre as partes são críticos para o estabelecimento de tal fidelidade. Só com a perfeita sintonia entre a solicitação dos consumidores, a administração dos pedidos, o estoque e a administração financeira se pode garantir a qualidade de serviço que sustente a fidelidade do consumidor.

Essa sintonia não se restringe ao ambiente interno da organização que fornece os serviços e produtos. Ela exige também o mesmo nível de integração da organização com seus parceiros. Para ilustrar a importância da integração entre diferentes companhias, basta ressaltar que as empresas distribuidoras de bens físicos são essenciais no cenário do comércio eletrônico (Lappin, 1996). O fluxo de bens físicos deve-se tornar tão eficiente quanto o fluxo de informação, para que os consumidores percebam as vantagens da utilização do comércio on-line.

A exploração das vantagens dos sistemas de comércio eletrônico exigirá grande esforço por parte das organizações que nele se envolverem. O desenvolvimento e implementação de tais sistemas demandará investimentos em recursos humanos com visão estratégica, conhecimento dos processos de negócio, dos sistemas herdados, competência tecnológica e habilidades gráficas. A capacidade de gerenciar equipes com habilidades multifuncionais e a definição do que deve ou não ser terceirizado serão elementos críticos. 
Se, por um lado, o ambiente da Web pode permitir baixo custo de entrada para uma empresa disposta a investir no comércio eletrônico, por outro lado manter os sistemas funcionando vai exigir um fluxo de recursos que deve ser levado em conta. Não é sem razão que muitos empreendedores iniciais estão sendo adquiridos por grandes empresas, como forma de suprir esta necessidade de recursos.

A presença de grandes empresas e a competição pela atenção do consumidor estão levando a uma exploração cada vez maior de tecnologia para aumentar a sofisticação nos recursos interativos por meio de gráficos, sons etc. Este incremento na qualidade dos sistemas leva ao aumento da complexidade de seu desenvolvimento e manutenção, conseqüentemente demandando maior quantidade de recursos financeiros e humanos, o que exige da organização um compromisso que só se justifica se for recompensado com o aumento das receitas.

Além das dificuldades tecnológicas, culturais e organizacionais, algumas outras barreiras se colocam no caminho do desenvolvimento do comércio eletrônico. Essas outras barreiras têm caráter estrutural, por estarem relacionadas ao desenvolvimento de instrumentos institucionais que viabilizem ampla utilização do comércio on-line.

\section{Barreiras Estruturais}

O ambiente próximo da anarquia, em que se desenvolveu a Internet, foi altamente positivo para a troca de informações e para o desenvolvimento de relações entre pessoas de todos os cantos do planeta. A comunidade de usuários da Internet cresceu sob regras de conduta não explicitadas, mas que ajudavam a controlar a participação dos usuários, num ambiente sem Governo centralizado; mas as regras informais, que ainda hoje predominam na Internet, não são suficientes para tocar negócios on-line.

A adoção da Internet como veículo de massa vai exigir dispositivos de regulamentação mais efetivos e explícitos, para propiciar a proliferação de transações comerciais. Existe uma relação inversa entre a confiança estabelecida entre os participantes de uma transação e o custo dela. O comércio eletrônico, para a sua consolidação, precisa garantir a realização de transações a custo compatível com as formas tradicionais de negociação.

Assim, é de se esperar que o crescimento do comércio eletrônico reforce a necessidade de definições de direitos de propriedade mais adequados à distribuição digital de informações, crie formas de trocas monetárias seguras e fáceis de serem utilizadas, e ainda ofereça garantias para localizar e punir os violadores das regras estabelecidas para o funcionamento do mundo on-line (Spar e Bussgang, 
1996). O comércio via Internet pode ser frustrante, caso sistemas de regulamentação de transações não se desenvolvam no mesmo ritmo da tecnologia que as possibilita.

Se a informação é a mercadoria mais fácil de ser comercializada eletronicamente, é também a que sofre mais riscos de ser apropriada e adulterada, sem qualquer tipo de controle. Se o ambiente de livre troca de informações pode ser excitante pelo lado de garantir, teoricamente, o democrático acesso de tudo a todos, a regra dos negócios é a transferência do direito sobre uma determinada propriedade, com a respectiva aquisição dos benefícios relativos a essa transferência por parte de quem a cede.

Para resolver o problema do direito à propriedade no mundo on-line, a alternativa mais lógica seria a adoção de mecanismos já existentes de copyright, o que garantiria a proprietários de produtos intangíveis, como idéias ou expressões, o direito de exploração comercial de sua mercadoria. Mais que as ambigüidades e incertezas inerentes à interpretação do que seja de fato o direito à propriedade sobre uma informação, no caso da Internet se fala de comercialização em um mercado global, o que significa que essas leis devem ser universalmente aceitas; isso dificulta ainda mais a possibilidade de consenso. A falta do estabelecimento de tais regras comuns para toda a comunidade on-line pode significar que menos empresas se interessem por transacionar seus produtos, uma vez que não podem ter garantidos os benefícios de seu investimento, por meio de troca que considerem justa.

Acordados os direitos de transferência de propriedade, as transações de negócio terão sucesso apenas se as trocas financeiras entre compradores e vendedores ocorrerem de forma simples, segura, barata e universalmente aceita. Há que se considerarem os riscos e os custos inerentes aos diversos meios de pagamento disponíveis mediante a Internet. Sistemas de pagamento baseados em mecanismos tradicionais têm sido adotados; novos formatos, como o dinheiro eletrônico ( $e$ cash), estão sendo discutidos; mas ainda é preciso encontrar um sistema que seja amplamente aceito.

Há duas formas tradicionais de pagamento que podem ser também utilizadas para o comércio eletrônico: as transações financeiras feitas por fora da Internet e os pagamentos com cartão de crédito. No primeiro caso, o comprador transfere uma quantidade de fundos de sua conta bancária para a conta do vendedor. Esta forma é muito demorada e cara para os padrões do comércio eletrônico, principalmente se consideradas as transações entre diferentes países. Embora possa ser viável para relacionamentos estáveis entre comprador e vendedor, para quantidades pequenas de pagamento ou para um comprador que faz compras eventuais de fontes diversas, 
este mecanismo não traz grandes vantagens na utilização do comércio eletrônico.

Para reduzir a morosidade e o custo das transações bancárias, o cartão de crédito pode ser utilizado para os pagamentos pela Internet. Mesmo utilizando-se de sistemas de encriptação, o que em tese limitaria o risco do comprador enviar os dados do cartão pela Internet, outros fatores devem ser considerados. Um deles é o custo da transação via cartão, que pode inviabilizar o desenvolvimento de novos modelos de negócio on-line, que dependam de transações baseadas em micropagamentos, por exemplo, jornais pagos-por-artigo.

A necessidade de sistemas de pagamento mais especializados levou à proposição do e-cash (Wayner, 1996). Por meio de um software próprio, um consumidor pode transferir certo valor monetário do banco para o seu computador, ou para um cartão específico, armazenando assim certa quantidade de $\boldsymbol{e}$-cash, como se tivesse uma carteira eletrônica. Transferindo parcelas do valor armazenado na sua carteira para o vendedor dos serviços que quer adquirir, o consumidor assim teria o direito de escolher: ou pagar em dinheiro eletrônico, garantindo a sua privacidade, mediante a encriptação, ou dispondo de valores tão pequenos, quanto fosse necessário, a um custo relativamente baixo por transação.

Além de necessitar de segurança adicional para evitar que o dinheiro seja roubado do computador do consumidor, muitas questões relativas à regulamentação da circulação de tal tipo de moeda teriam de ser consideradas. Por exemplo, como evitar a falsificação? Como seria controlada a emissão e a circulação do $e$-cash? E que influência a circulação do dinheiro eletrônico teria sobre o controle que os Governos exercem sobre as economias nacionais atualmente (Frezza, 1996)?

Os instrumentos de perseguição são mais precários ainda. Se os invasores de computadores são ousados o suficiente para entrar - e alterar - sites de agências de segurança e de corporações militares sem ser identificados, que garantia um consumidor comum terá de que, caso seja roubado eletronicamente, os organismos de repressão à criminalidade terão condições de identificar o criminoso? E mesmo que ele seja identificado, a sua punição vai depender de possíveis acordos entre os países envolvidos.

Empresas privadas estão investindo para conseguir instaurar a segurança e conquistar a confiança que pode ajudar a desenvolver o comércio eletrônico; mas a inexistência de terceiros isentos, e com força para arbitrar pendências, pode retardar o avanço do comércio eletrônico, pois o interesse de empresas comerciais pode conflitar com o dos consumidores.

O comprometimento dos bancos (Andersen, 1998), por exemplo, também é vital para a consolidação do comércio on-line; mas as incertezas sobre como lidar 
com os problemas relativos ao trânsito de valores pela Internet ainda deixam no ar as reais possibilidades do comércio eletrônico, para organizações que transacionam no mercado financeiro.

Assim, a falta de balizamento em questões estruturais e na criação de mecanismos regulatórios representa séria barreira ao desenvolvimento do comércio eletrônico. Se comunidades privadas, constituídas eletronicamente, terão força para manter o nível de regulação necessário, para que as atividades comerciais possam fluir através da Web, como alguns sugerem (Spar e Bussgang, 1996), não é possível prever ainda.

O papel dos Estados nacionais e de outras instituições reguladoras permanece obscuro dentro do universo do comércio eletrônico. A falta intrínseca de controle e a impossibilidade de Governos nacionais intervirem, para oferecer níveis de estabilidade e segurança condizentes, pode representar grande obstáculo ao desenvolvimento das atividades comerciais pela Internet.

\section{CONCLUSÕES}

A evolução da tecnologia, particularmente a convergência entre a computação e a telecomunicação (Messerschmitt,1996), e a sua disseminação estão revolucionando a natureza das comunicações num sentido amplo; pela importância que as comunicações têm na sociedade, impactos muito profundos ainda estão por ocorrer.

Na prática, a Internet criou uma revolução nos meios de comunicação global; está alterando dramaticamente as possibilidades de se transacionar comercialmente em todo o mundo. A Web está cada dia se consolidando como o canal mais eficiente de interligação entre empresas e consumidores, sejam eles indivíduos ou outras organizações.

As possibilidades de se acrescentar valor com a utilização da Internet existem e estão sendo exploradas por empresas pioneiras. O comércio eletrônico já existe na prática e está em expansão, embora a aceitação ampla do mercado on-line necessite de pesquisas que indiquem com melhor precisão a sua evolução.

Empresas já estão ganhando dinheiro com a Internet (Bussiness Week, 1996). Nesse grupo estão aquelas que fornecem produtos e serviços essenciais à entrada no mundo on-line, ou seja, as empresas que atuam nos mercados de telefonia e computação. Nesse grupo está também uma grande massa de outras empresas, que ajudam a consolidar a estrutura da Web, necessária para o funcionamento do 
comércio eletrônico. Provedores de acesso - Internet Service Providers (ISP), desenvolvedores de softwares para navegação (browsers), designers de páginas Web, administradores de ferramentas de busca etc, estão entre essas empresas.

Essas empresas estão entre as primeiras a apresentar alternativas para explorar, da melhor forma possível, as vantagens do comércio eletrônico. É com essas alternativas que as demais empresas, desejosas de explorar comercialmente o espaço virtual, criado pela Intenet, seja através de anúncios, da venda de assinaturas, seja de transações diretas, vão poder consolidar o comércio eletrônico.

A velocidade e o alcance dessa consolidação dependem da superação de obstáculos de vários tipos. Existem obstáculos tecnológicos, como a dificuldade para desenvolvimento de ferramentas adequadas à transação on-line; culturais, como a modificação de hábitos de consumo; organizacionais, pela adaptação das empresas ao novo ambiente e até mesmo a superação dos obstáculos estruturais da sociedade, o que pode criar condições para que predomine um ambiente de confiança, necessário para o desenvolvimento do comércio eletrônico.

\section{ReferênCias BiblográficAs}

\section{ANDERSEN, B. K.}

It's time for banks to dive into ecommerce. The New York Times, May 261998.

BEAM, C.;

SEGEV, A.

The rise of electronic commerce : contributions from three factors. CITM Working Paper, Aug. 1996.

BLOCH, M.;

PIGNEUR, Y.;

SEGEV, A.

On the road of electronic commerce : a business value framework, gaining competitive advantage and some research issues. CITM Working Paper, Mar. 1996.

BUSINESS WEEK.

Making money on the net. [S.1.], 23 Sept. 1996.

ELETRONIC FROUNTIER FOUNDATION

[online] Disponível na Internet via WWW.URL: http://www.eff.org.

\section{ETRUST.}

[online] Disponível na Internet via WWW.UR:http://www.etrust.org. 


\section{FREZZA, B. A.}

The internet and the end of monetary sovereignty. [online] Disponível na Internet via W W W. U R L : h t t p : / / www.cato.org/moneyconf/14mc4.html. 14TH ANNUAL MONETARY CONFERENCE (1996 : Washington, D.C.). Proceedings... Washington, D.C.: Cato Institutes, 1996.

GUPTA, A.;

STAHL, D. O.;

WHINSTON, A. B.

A stochastic equilibrium model of internet pricing. In: INDUSTRIAL ORGANIZATIONAL SEMINAR (1996 : Berkeley). Proceedings... Berkeley : Agricultural Research and Economics Department, 1996.

HOFFMAN, D. L.;

NOVAK, T. P.;

CHATTERJEE, $\mathrm{P}$.

Commercial scenarios for the web : opportunities and challenges. [online] Disponível na Internet via WWW.URL : http:// www.usc.edu/dept/annenberg/ vol1/issue $3 /$ hoffman.html. Journal of Computer-Mediated Communication, v. 1, n. 3, Dec. 1995.
KLINE, D.

False alarm : credit card security. Wired, Oct. 1995.

LAPPIN, T.

The airline of the internet. Wired, Dec. 1996.

\section{LEVY, S.}

Wisecrackers. Wired, Mar. 1996.

MESSERSCHMITT, D. G.

The convergence of telecommunications and computing: what are the implications today? In: IEEE (1996 : [S.1.]). Proceedings... [S.1. : s.n.], 1996.

NEGROPONTE, N.

Being digital. [s.n.] : A. Knopf, 1995.

SEGEV, A. et al.

Financial EDI over the internet: a case study. CITM Working Paper, June 1995.

SOKOL, P.

EDI : the competitive edge. New York : McGraw-Hill, 1989.

SPAR, D.;

BUSSGANG, J. J.

Ruling the net Harvard Business

Review, May/June 1996. 
STEINFIELD, C.;

KRAUT, R.;

PLUMMER, A.

The impact of eletronic commerce on buyer-seller relationships. [online] Disponível na Internet via WWW.URL : http:// www.usc.edu/dept/annerberg/ vol1/issue $3 /$ steinfld.html. Journal of Computer-Mediated Communication, v. 1, n. 3, Dec. 1995.

\section{TELECOM}

Finland starts internet telephone services. [online] Disponível na
Internet via WWW.URL : http:// www.tfi.net/press/phone/html. Press Release, 10 Dec. 1996.

THE NEW YORK TIMES.

FCC adopts plan offering cheap link to internet. Jan. 91997.

WARE, J. P. et al.

Search for digital excellence. New York : McGraw-Hill, 1998.

WAYNER, P.

Digital cash : commerce on the net. 2. ed. London : Academic Press, 1996. 